\title{
REINFORCEMENT LEARNING FOR ROBOT CONTROL USING PROBABILITY DENSITY ESTIMATIONS
}

\author{
Alejandro Agostini and Enric Celaya \\ Institut de robòtica i Informàtica Industrial, (CSIC-UPC), Barcelona, Spain \\ \{agostini, celaya\}@iri.upc.edu
}

Keywords: Machine Learning in control applications, Reinforcement learning.

\begin{abstract}
The successful application of Reinforcement Learning (RL) techniques to robot control is limited by the fact that, in most robotic tasks, the state and action spaces are continuous, multidimensional, and in essence, too large for conventional RL algorithms to work. The well known curse of dimensionality makes infeasible using a tabular representation of the value function, which is the classical approach that provides convergence guarantees. When a function approximation technique is used to generalize among similar states, the convergence of the algorithm is compromised, since updates unavoidably affect an extended region of the domain, that is, some situations are modified in a way that has not been really experienced, and the update may degrade the approximation. We propose a RL algorithm that uses a probability density estimation in the joint space of states, actions and $Q$-values as a means of function approximation. This allows us to devise an updating approach that, taking into account the local sampling density, avoids an excessive modification of the approximation far from the observed sample.
\end{abstract}

\section{INTRODUCTION}

Any robotic application requires a precise control of the robot effectors. In many situations, the design of the control system and the necessary tuning of its parameters becomes a hard task, and there is a great interest in providing the robot with the ability to learn by itself how to control its effectors through experience. The most usual approach to achieve this is Reinforcement Learning (RL) (Sutton and Barto, 1998), in which the robot must find an optimal action policy by trial and error using a signal, called reinforcement, that tells how good was the result obtained after each executed action.

Reinforcement Learning algorithms, like value iteration or policy iteration, are known to converge in the case of finite state-action spaces, where a tabular representation of the value function or the utility function $Q$ can be used. However, in a typical robot control problem, the state and action spaces are continuous or too large, so that it is necessary to use some form of function approximation. In this case convergence is no longer granted in general, and this is attributed to the fact that the update on the function approximation that takes place after each experience is not local to the precise observed point in the state-action space, but influences the values in other regions, possibly undoing the learning done so far (Riedmiller, 2005a). This problem is magnified when the sampling of the domain is too biased, causing some regions to be updated much more oftenly than others.

Recently, much work has been done in RL with function approximation. For example, (Ernst et al., 2005 ) proposed the fitted $Q$ Iteration algorithm, based on previous work of (Ormoneit and Sen, 2002) on kernel-based RL, and using randomized trees for function approximation. Similarly, (Riedmiller, 2005a) proposed the Neural Fitted $Q$ Iteration (NFQ) algorithm, which uses a multi-layer perceptron for function representation. In a different approach, (Rasmussen and Kuss, 2004) used Gaussian Processes (GP) to model the system dynamics, and a further GP to represent the Value function. The same approach is followed by (Rottmann and Burgard, 2009), while (Engel et al., 2005) used a GP to directly represent the Q-function in a model-free setting. All these algorithms fall into the class of the so-called fitted value iteration algorithms (Gordon, 1995), which, in order to approximate the desired function, take a finite number of training samples and try to fit the function to them in a batch, iterative process. The usual approach 
to obtain the training samples, when the knowledge of the system dynamics is available, consists in sampling uniformly all the state-action space to build a training set that covers all possible situations sufficiently well. Clearly, this procedure is not possible when dealing with a real system with unknown dynamics, in what case samples can only be observed while interacting with the real system. In the simplest cases, it is possible to roughly cover the whole state space by chaining a number of random actions, as in (Ernst et al., 2005). However, when the problem grows in complexity, the probability of executing a random sequence that drives the system to the interesting regions of the workspace may be too low to be achieved in practical time. In such cases it is necessary to exploit the knowledge already obtained with previous interactions (Riedmiller, 2005a; Ernst et al., 2005).

It has to be noted that the need of exploiting what has been learned so far introduces a tendency to experience the most promising states much more oftenly than others, and this systematically produces a very biased sampling that aggravates the perturbing effect caused by non-local updating pointed out before. In (Riedmiller, 2005a), this problem is avoided by assuring that all datapoints are used for update the same number of times. This is made possible by remembering a dense enough set of transitions and performing full updates in batch mode. In fact this is a common trait of all fitted value iteration algorithms. From a computational point of view, this approach is very computationally intensive, since all datapoints are used a large number of times until convergence is reached. A more efficient approach would result if, instead of retraining with old data in batch, an incremental updating could be achieved in which the perturbing effect of new samples on old estimations was attenuated.

In the present work, we address the problem of the biased sampling with incremental updating. In our approach, we take into account how often each region of the domain has been visited, updating more locally those regions that are more densely sampled. To do this, we need an estimation of the sampling density, for what we use a Gaussian Mixture Model (GMM) representing a probability density of samples in the joint space of states, actions, and $Q$-values. At the same time, this density estimation can be used as a means of function approximation for the $Q$-function. Density estimations are receiving increasing interest in the field of machine learning (Bishop, 2006), since they keep all the information contained in the data, that is, they provide estimations not only for the expected function value, but also for its uncertainty.
Despite density estimations are more demanding than simple function approximation (due to the fact that they embody more information), their use for function approximation has been advocated by different authors (Figueiredo, 2000; Ghahramani and Jordan, 1994), noting that simple and well understood tools like the Expectation-Maximization (EM) algorithm (Dempster et al., 1977) can be used to obtain accurate estimations of the density function.

The rest of the paper is organized as follows: Section 2 briefly resumes the basics of RL. Section 3 introduces the concepts of GMM for multivariate density estimation, and the EM algorithm in its batch version. In Section 4 we define the on-line EM algorithm for the GMM. In Section 5, we present our approach to deal with biased sampling. In Section 6 we develop our RL algorithm using density estimation of the $Q$ value function, involving action evaluation and action selection. Section 7 describes the test control application to show the feasibility of the approach. We conclude in Section 8 with a discussion of the proposed approach.

\section{THE REINFORCEMENT LEARNING PARADIGM}

In the RL paradigm, an agent must improve its performance by selecting actions that maximize the accumulation of rewards provided by the environment (Sutton and Barto, 1998). At each time step, the agent observes the current state $s_{t}$ and chooses an action $a_{t}$ according to its policy $a=\pi(s)$. The environment changes to state $s_{t+1}$ in response to this action, and produces an instantaneous reward $r\left(s_{t}, a_{t}\right)$. The agent must experiment by interacting with the environment in order to find the optimal action policy from the outcome of its past experiences. One of the most popular algorithms used in RL is $Q$-Learning (Watkins and Dayan, 1992), which uses an action-value function $Q(s, a)$ to estimate the maximum expected future cumulative reward that can be obtained by executing action $a$ in situation $s$ and acting optimally thereafter. $Q$-learning uses a sampled version of the Bellman optimality equations (Bellman and Dreyfus, 1962) to estimate instantaneous $q$ values,

$$
q\left(s_{t}, a_{t}\right)=r\left(s_{t}, a_{t}\right)+\gamma \max _{a} Q\left(s_{t+1}, a\right)
$$

where $\max _{a} Q\left(s_{t+1}, a\right)$ is the estimated maximum cumulative reward corresponding to the next observed situation $s_{t+1}$, and $\gamma$ is a discount factor, with values in $[0,1]$ that regulates the importance of future rewards with respect to immediate ones. At a given stage of 
the learning, the temporary policy can be derived from the estimated $Q$-function as,

$$
\pi(s)=\underset{a}{\operatorname{argmax}} Q(s, a)
$$

In actor/critic architectures, a policy function (called the actor) is learned and explicitly stored, so that actions are directly decided by the actor and do not need to be computed from the maximization in (2). Despite this computational advantage, the learning of an actor may slow down convergence, since then the learning of the $Q$-function must be done onpolicy instead of off-policy, and both functions, actor and critic, must adapt to each other to reach convergence. In our implementation we avoid the use of an actor, and thus we must face the problem of maximizing the $Q(s, a)$ function in (2).

The basic formulation of $Q$-learning assumes discrete state-action spaces and the $Q$-function is stored in a tabular representation. For continuous domains a function approximation is required to represent the $Q$-function and generalize among similar situations. In next sections we present our proposal for function approximation using density estimations.

\section{DENSITY ESTIMATION WITH A GAUSSIAN MIXTURE MODEL}

A Gaussian Mixture Model (Bishop, 2006) is a weighted sum of multivariate Gaussian probability density functions, and is used to represent general probability density distributions in multidimensional spaces. It is assumed that the samples of the distribution to be represented have been generated through the following process: first, one Gaussian is randomly selected with a priori given probabilities, and then, a sample is randomly generated with the probability distribution of the selected Gaussian. According to this, the probability density function of generating sample $\mathbf{x}$ is:

$$
p(\mathbf{x} ; \Theta)=\sum_{i=1}^{K} \alpha_{i} \mathcal{G}\left(\mathbf{x} ; \mu_{i}, \Sigma_{i}\right)
$$

where $K$ is the number of Gaussians of the mixture; $\alpha_{i}$, usually denoted as the mixing parameter, is the prior probability, $P(i)$, of Gaussian $i$ to generate a sample; $\mathcal{G}\left(\mathbf{x} ; \mu_{i}, \Sigma_{i}\right)$ is a multidimensional normal Gaussian function with mean vector $\mu_{i}$ and covariance matrix $\Sigma_{i}$; and $\Theta=\left\{\left\{\alpha_{1}, \mu_{1}, \Sigma_{1}\right\}, \ldots,\left\{\alpha_{K}, \mu_{K}, \Sigma_{K}\right\}\right\}$ is the whole set of parameters of the mixture. By allowing the adaption of the number $K$ of Gaussians in the mixture, any smooth density distribution can be approximated arbitrarily close (Figueiredo, 2000). The parameters of the model can be estimated using a maximum-likelihood estimator (MLE). Given a set of samples $\mathbf{X}=\left\{\mathbf{x}_{t} ; t=1, \ldots, N\right\}$, the likelihood function is given by

$$
\mathcal{L}[\mathbf{X} ; \Theta]=\prod_{t=1}^{N} p\left(\mathbf{x}_{t} ; \Theta\right) .
$$

The maximum-likelihood estimation of the model parameters is the $\Theta$ that maximizes the likelihood (4) for the data set $\mathbf{X}$. Direct computation of the MLE requires complete information about which mixture component generated which instance. Since this information is missing, the EM algorithm, described in the next section, is often used.

\subsection{The Expectation-maximization Algorithm}

The Expectation-Maximization (EM) algorithm (Dempster et al., 1977) is a general tool that permits to estimate the parameters that maximize the likelihood function (4) for a board class of problems when there are some missing data. The EM method first produces an estimation of the expected values of the missing data using initial values of the parameters to be estimated (E step), and then computes the MLE of the parameters given the expected values of the missing data ( $M$ step). This process is repeated iteratively until a convergence criterion is fulfilled.

In this section we briefly describe how EM is applied to the specific case of a GMM. The process starts with an initialization of the mean vectors and covariance matrices of the Gaussians. The E step consists in obtaining the probability $P\left(i \mid \mathbf{x}_{t}\right)$ for each component $i$ of generating instance $\mathbf{x}_{t}$, that we denote by $w_{t, i}$

$$
w_{t, i}=P\left(i \mid \mathbf{x}_{t}\right)=\frac{P(i) p\left(\mathbf{x}_{t} \mid i\right)}{\sum_{j=1}^{K} P(j) p\left(\mathbf{x}_{t} \mid j\right)}=\frac{\alpha_{i} \mathcal{G}\left(\mathbf{x}_{t} ; \mu_{i}, \Sigma_{i}\right)}{\sum_{j=1}^{K} \alpha_{j} \mathcal{G}\left(\mathbf{x}_{t} ; \mu_{j}, \Sigma_{j}\right)}
$$

where $t=1, . ., N$ and $i=1, \ldots, K$. The maximization step consists in computing the MLE using the estimated $w_{t, i}$. It can be shown (Duda et al., 2001) that, for the case of a GMM, the mixing parameters, means, and covariances are given by

$$
\begin{aligned}
& \alpha_{i}=\frac{1}{N} \sum_{t=1}^{N} w_{t, i} \\
& \mu_{i}=\frac{\sum_{t=1}^{N} w_{t, i} \mathbf{x}_{t}}{\sum_{t=1}^{N} w_{t, i}}
\end{aligned}
$$




$$
\Sigma_{i}=\frac{\sum_{t=1}^{N} w_{t, i}\left(\mathbf{x}_{t}-\mu_{i}\right)\left(\mathbf{x}_{t}-\mu_{i}\right)^{\top}}{\sum_{t=1}^{N} w_{t, i}}
$$

\section{ON-LINE EM}

Estimating a probability density distribution by means of the EM algorithm involves the iteration of $\mathrm{E}$ and $\mathrm{M}$ steps on the complete set of available data, that is, the mode of operation of EM is in batch. However, in $Q$-learning, sample data are not all available at once: they arrive sequentially and must be used online to improve the policy that will allow an efficient exploration-exploitation strategy. This prevents the use of the batch EM algorithm, and requires an on-line, incremental version of it. Several incremental EM algorithms have been proposed for the Gaussian Mixture Model applied to clustering or classification of stationary data (Song and Wang, 2005; Arandjelovic and Cipolla, 2005).

The approach proposed in (Song and Wang, 2005) in not strictly an on-line EM algorithm. It applies the conventional batch EM algorithm onto separate data streams corresponding to successive episodes. For each new stream, a new GMM model is trained in batch mode and then merged with the previous model. The number of components for each new GMM is defined using the Bayesian Information Criterion, and the merging process involves similarity comparisons between Gaussians. This method involves many computationally expensive processes at each episode and tends to generate more components than actually needed. The applicability of this method to RL seems limited, not only for its computational cost, but also because, due to the non-stationarity of the $Q$-estimation, old data should not be taken as equally valid during all the process.

The work of (Arandjelovic and Cipolla, 2005) performs incremental updating of the density model using no historical data and assuming that consecutive data vary smoothly. The method maintains two GMMs: the current GMM estimation, and a previous GMM of the same complexity after which no model updating (i.e. no change in the number of Gaussians) has been done. By comparing the current GMM with the historical one, it is determined if new Gaussians are generated or if some Gaussians are merged together. Two observed shortcomings of the algorithm are that the system fails when new data is well explained by the historical GMM, and when consecutive data violate the condition of smooth variation.

In (Sato and Ishii, 2000), an on-line EM algorithm is presented for the Normalized Gaussian Network (NGnet), a model closely related to the GMM. This algorithm is based on the works of (Nowlan, 1991; Neal and Hinton, 1998). In (Nowlan, 1991) a method for the incremental adaptation of the model parameters using a forgetting factor and cumulative statistics is proposed, while in (Neal and Hinton, 1998) the method in (Nowlan, 1991) is evaluated and contrasted with an incremental version which performs steps of EM over a fixed set of samples in an incremental way. The method proposed in (Sato and Ishii, 2000) uses foundations of both works to elaborate an on-line learning algorithm to train a NGnet for regression, where weighted averages of the model parameters are calculated using a learning rate that implicitly incorporates a forgetting factor to deal with nonstationarities. Inspired by this work, we developed an on-line EM algorithm for the GMM. Our approach uses cumulative statistics whose updating involves a forgetting factor explicitly.

\subsection{On-line EM for the GMM}

In the on-line EM approach, an E step and an M step are performed after the observation of each individual sample. The E step does not differ from the batch version (equation (5)), except that it is only computed for the new sample. For the M step, the parameters of all mixture components are updated with the new sample. For this, we define the following time-discounted weighted sums

$$
\begin{aligned}
W_{t, i} & =[[1]]_{t, i} \\
X_{t, i} & =[[\mathbf{x}]]_{t, i} \\
(X X)_{t, i} & =\left[\left[\left(\mathbf{x}-\mu_{i}\right)\left(\mathbf{x}-\mu_{i}\right)^{\top}\right]\right]_{t, i}
\end{aligned}
$$

where we use the notation:

$$
[[f]]_{t, i}=\sum_{\tau=1}^{t}\left(\prod_{s=\tau+1}^{t} \lambda_{s}\right) f_{\tau, i} w_{\tau, i}
$$

where $\lambda_{t}$, which ranges in $[0,1]$, is a time dependent discount factor introduced for forgetting the effect of old, possibly outdated values. Observe that for low values of $\lambda_{t}$, the influence of old data decreases progressively, so that they are forgotten along time. This forgetting effect of old data is attenuated when $\lambda_{t}$ approaches 1: in this case, old and new data have the same influence in the sum. As learning proceeds and data values become more stable, forgetting them is no more required and $\lambda_{t}$ can be made to progressively approach 1 to reach convergence.

The discounted sum $W_{t, i}$ can be interpreted as the accumulated number of samples (composed of fractions $w_{t, i}$ ) attributed to unit $i$ along time, with forgetting. Similarly, $X_{t, i}$ corresponds to the accumulated 
sum with forgetting of sample vectors $\mathbf{x}$ attributed to unit $i$, which is used to derive the mean vector $\mu_{i}$. In the same way, $(X X)_{t, i}$ accumulates the matrices of covariances of the vectors $\mathbf{x}$ attributed to unit $i$, which are used to calculate the covariance matrix $\Sigma_{i}$.

From (12), we obtain the recursive formula:

$$
[[f]]_{t, i}=\lambda_{t}[[f]]_{t-1, i}+f_{t, i} w_{t, i}
$$

When a new sample $\mathbf{x}_{t}$ arrives, all the accumulators are updated with the incremental formula (13), and new estimators for the GMM parameters are obtained as:

$$
\begin{gathered}
\alpha_{t, i}=\frac{W_{t, i}}{\sum_{j=1}^{K} W_{t, i}} \\
\mu_{t, i}=\frac{X_{t, i}}{W_{t, i}} \\
\Sigma_{t, i}=\frac{(X X)_{t, i}}{W_{t, i}} .
\end{gathered}
$$

The approximation capabilities of a GMM depend on the number $K$ of Gaussians of the mixture. Since we can not determine the most appropriate number beforehand, the number of Gaussians can be incremented on-line. We delay the explanation of the process for unit generation until Section 6.2.

\section{DEALING WITH BIASED SAMPLING}

In the incremental formula (13), the factor $\lambda_{t}$ is used to progressively replace (forget) old data by new arrived ones in a smooth way. This is the desired effect when data are presented in a statistically unbiased way, so that all past entries are equally forgotten at the arrival of each new sample. However, in RL, data are not uniformly distributed on the state-action space, but are obtained along the trajectories followed by the agent, and are particularly biased toward the good-valued regions due to the need for exploitation. That is, convergence regions are more frequently sampled, causing their densities to increase, but this is at the expense of lowering densities and forgetting data in other regions. This is undesirable since, statistics in regions with low $Q$ values, and hence sparsely sampled, will get their data lost. This can be seen by setting $w_{t, i}=0$ in equation (13), what yields:

$$
[[f]]_{t, i}=\lambda_{t}[[f]]_{t-1, i},
$$

showing that the accumulators of units that are seldom activated will decay to 0 . To avoid this we modify the updating formula (13) in this way,

$$
[[f]]_{t, i}=\lambda_{t}^{w_{t, i}}[[f]]_{t-1, i}+f_{t, i} w_{t, i}
$$

In this updating formula, the power $w_{t, i}$ prevents undesired changes in the parameters of the Gaussians which are not responsible of generating the observed values. Thus, if we make $w_{t, i}=0$ in (18), what we get is:

$$
[[f]]_{t, i}=[[f]]_{t-1, i}
$$

so that the values of the statistics of the inactive units remain unchanged. On the other hand, in the limit case of $w_{t, i}=1$, corresponding to a full activation of unit $i$, the effect of the new updating formula is the same as before:

$$
[[f]]_{t, i}=\lambda_{t}[[f]]_{t-1, i}+f_{t, i}
$$

Therefore, we will adopt the updating formula (18) that keeps better track of less explored regions.

\section{$6 \quad Q$-LEARNING WITH A GMM}

In the case of $Q$-Learning, samples are of the form $\mathbf{x}_{t}=\left(\mathbf{s}_{t}, a_{t}, q\left(\mathbf{s}_{t}, a_{t}\right)\right)$, consisting of the visited state $\mathbf{s}_{t}$, the executed action $a_{t}$, and the estimated value of $q\left(\mathbf{s}_{t}, a_{t}\right)$ as given by eq. (1). To obtain this estimation we need to evaluate $\max Q\left(\mathbf{s}_{t+1}, a\right)$, where $Q(\mathbf{s}, a)$ is defined as the expected value of $q$ given $\mathbf{s}$ and $a$ for the joint probability distribution provided by the GMM:

$$
\begin{gathered}
p(\mathbf{s}, a, q)=\sum_{i=1}^{K} \alpha_{i} \mathcal{G}\left(\mathbf{s}, a, q ; \mu_{i}, \Sigma_{i}\right), \\
Q(\mathbf{s}, a)=E[q \mid \mathbf{s}, a]=\mu(q \mid \mathbf{s}, a)
\end{gathered}
$$

To compute this, we must first obtain the distribution $p(q \mid \mathbf{s}, a)$. Decomposing the covariances $\Sigma_{i}$ and means $\mu_{i}$ in the following way:

$$
\begin{gathered}
\mu_{i}=\left(\begin{array}{c}
\mu_{i}^{(\mathbf{s}, a)} \\
\mu_{i}^{q}
\end{array}\right) \\
\Sigma_{i}=\left(\begin{array}{ll}
\Sigma_{i}^{(\mathbf{s}, a)(\mathbf{s}, a)} & \Sigma_{i}^{(\mathbf{s}, a) q} \\
\Sigma_{i}^{q(\mathbf{s}, a)} & \Sigma_{i}^{q q}
\end{array}\right),
\end{gathered}
$$

the probability distribution of $q$, for the given state $\mathbf{s}$ and a tentative action $a$, can then be expressed as:

$$
p(q \mid \mathbf{s}, a)=\sum_{i=1}^{K} \beta_{i}(\mathbf{s}, a) \mathcal{G}\left(q ; \mu_{i}(q \mid \mathbf{s}, a), \sigma_{i}(q)\right)
$$

where,

$$
\begin{array}{r}
\mu_{i}(q \mid \mathbf{s}, a)=\mu_{i}^{q}+\Sigma_{i}^{q(\mathbf{s}, a)}\left(\Sigma_{i}^{(\mathbf{s}, a)(\mathbf{s}, a)}\right)^{-1}\left((\mathbf{s}, a)-\mu_{i}^{(\mathbf{s}, a)}\right) \\
\sigma_{i}^{2}(q)=\Sigma_{i}^{q q}-\Sigma_{i}^{q(\mathbf{s}, a)}\left(\Sigma_{i}^{(\mathbf{s}, a)(\mathbf{s}, a)}\right)^{-1} \Sigma_{i}^{(\mathbf{s}, a) q}
\end{array}
$$




$$
\beta_{i}(\mathbf{s}, a)=\frac{\alpha_{i} \mathcal{G}\left(\mathbf{s}, a ; \mu_{i}^{(\mathbf{s}, a)}, \Sigma_{i}^{(\mathbf{s}, a)(\mathbf{s}, a)}\right)}{\sum_{j=1}^{K} \alpha_{j} \mathcal{G}\left(\mathbf{s}, a ; \mu_{j}^{(\mathbf{s}, a)}, \Sigma_{j}^{(\mathbf{s}, a)(\mathbf{s}, a)}\right)} .
$$

From (25) we can obtain the conditional mean and variance, $\mu(q \mid \mathbf{s}, a)$ and $\sigma^{2}(q \mid \mathbf{s}, a)$, of the mixture at a point $(\mathbf{s}, a)$ as:

$$
\begin{gathered}
\mu(q \mid \mathbf{s}, a)=\sum_{i=1}^{K} \beta_{i}(\mathbf{s}, a) \mu_{i}(q \mid \mathbf{s}, a) \\
\sigma^{2}(q \mid \mathbf{s}, a)=\sum_{i=1}^{K} \beta_{i}(\mathbf{s}, a)\left(\sigma_{i}^{2}(q)+\left(\mu_{i}(q \mid \mathbf{s}, a)-\mu(q \mid \mathbf{s}, a)\right)^{2}\right)
\end{gathered}
$$

Equation (29) is the estimated $Q$ value for a given state and action, while (30) is its estimated variance. Our purpose was to find the action that maximizes $Q(\mathbf{s}, a)$. Unfortunately, this cannot be done analytically, but an approximated value can be obtained by numerical techniques. In our implementation, we take the approach of computing $Q(\mathbf{s}, a)$ for a finite number of actions, and taking the action that provides the largest $Q$ value as the approximated maximum.

\subsection{Action Selection}

If our purpose is to greedily exploit the policy learned so far, as defined in (2), we must execute the action $a$ corresponding to the maximum $Q(\mathbf{s}, a)$ value for the current state $\mathbf{s}$, obtained as just explained. However, during learning, exploratory actions are also necessary. This is the classical exploration/exploitation tradeoff. Instead of following an undirected exploration method (using the nomenclature of (Dearden et al., 1998)), we make use of the uncertainty in the estimation of the $Q$ values to balance the information gain obtained from exploration, with the reward gain of executing the optimal action. For this, to each evaluated action, we assign a $Q$ value obtained stochastically from a normal distribution with mean (29) and variance (30), and select the action that provided the highest value. This exploration strategy increases the probability of executing exploratory actions when predictions are less certain, but this probability decreases when the mean value is lower.

\subsection{Unit Generation}

Since the main purpose of our GMM is to represent the $Q$ function, the generation of new Gaussians is principally driven by the need to better approximate the set of observed $q$ values. Thus, a new Gaussian is generated when the two following conditions are satisfied: 1) The estimation error of the observed $q$ value is larger than a predefined value $\delta$, and 2) The sample density in the experienced instance is below a threshold $\rho$. These criteria are expressed as:

$$
(q(s, a)-\mu(q \mid \mathbf{s}, a))^{2} \geq \delta
$$

and

$$
p(\mathbf{s}, a, q)=\sum_{i=1}^{K} \alpha_{i} \mathcal{G}\left(\mathbf{s}, a, q ; \mu_{i}, \Sigma_{i}\right) \leq \rho
$$

Whenever both criteria are fulfilled, a Gaussian is generated with parameters given by

$$
\begin{gathered}
W_{K+1}=1 \\
\mu_{K+1}(\mathbf{s}, a, q)=\left(\mathbf{s}_{t}, a_{t}, q\left(\mathbf{s}_{t}, a_{t}\right)\right) \\
\Sigma_{K+1}=C \operatorname{diag}\left\{d_{1}, \ldots, d_{D}, d_{a}, d_{q}\right\},
\end{gathered}
$$

where $d_{i}$ is the total range size of variable $i$; $D$ is the dimension of the state space; and $C$ is a positive value defining the dispersion of the new Gaussian. The imposition of the second condition is to avoid a proliferation of Gaussians in densely sampled regions.

\section{EXPERIMENTS}

To demonstrate the performance of the method we apply it to the classical benchmark problem of swinging up and stabilizing an inverted pendulum with limited torque (Doya, 2000). The task consists in swinging the pendulum until reaching the upright position and then stay there indefinitely. The optimal policy for this problem is not trivial since, due to the limited torques available, the controller has to swing the pendulum several times until its kinetic energy is large enough to overcome the load torque and reach the upright position.

The state space is two-dimensional and is configured by the angular position $\theta$ and angular velocity $\dot{\theta}$. We take advantage of the symmetry of the problem by identifying states with inverted angular position and velocity: $(\theta, \dot{\theta}) \sim(-\theta,-\dot{\theta})$. The GMM for density estimation is defined in the four-dimensional joint space $\mathbf{x}=(\theta, \dot{\theta}, a, q)$. As the reward signal (or, in this case, the cost that we have to minimize) we simply take the absolute value of the angle of the pendulum from its top position: $\operatorname{cost}(\theta, \dot{\theta})=-r(\theta, \dot{\theta})=|\theta|$ which ranges in the interval $[0, \pi]$. The discount coefficient $\gamma$ in equation 1 is set to 0.85 .

The setting of our system consists in the following. We provide the system with 10 initial Gaussians. The elements of the mean $\mu_{i}$ of the mixture component $i$ are selected randomly, except for the $q$ variable that is initialized to the maximum possible value to 
favor exploration of unvisited regions. The initial covariance matrices $\Sigma_{i}$ are diagonal and the variance for each variable is set to one tenth of the total span of its range. Each Gaussian is initialized with an accumulated number of samples $W_{i}=0.1$. This small value makes the component $i$ to have little influence in the estimation while there is no, or little, updating.

The discount factor $\lambda_{t}$ for the computation of the discounted sums (Section 4) takes values from the equation

$$
\lambda_{t}=1-1 /(a t+b),
$$

where $b$ fixes the initial value of $\lambda_{t}$ and $a$ determines its growth rate toward 1 . In our experiments we set $a=0.001$, and the value of $b$ depends on whether the updating formula (13) or (18) is used. In the first case, $b=10$, and when (18) is used, $b=1000$ to compensate for the effect of the exponent $w_{t, i}<1$. For the only purpose of the following discussion, we will refer to updates performed according to (13) as simply local updating, while updates performed according to (18) as exponentially local updating.

We performed the experiments using episodes of 50 seconds with an actuation interval of 0.1 seconds. At the beginning of each episode, the pendulum is placed in the hang-down position. At the end of each episode, a test of 30 seconds is performed exploiting the policy learned so far. As the result of the test we take the sum of the costs obtained at each time step of $0.1 \mathrm{sec}$.

Figure 1 shows the performance of the learning system when using simply local updating. The graphic corresponds to the average of 50 independent experiments of 100 episodes each. The system shows a stable performance after about 60 episodes, and reaches a total accumulated cost of around 100 . The best performance obtained by exhaustive manual tuning was near 44 , and corresponds to the line at the lower part of the figure. The best result obtained by a single experiment test was 44.42 . In average, the total number of Gaussians at the end of the experiments was about 84 .

In order to compare these results with those of (Riedmiller, 2005b), we will take into account the total number of times the system needs to be updated with a sample to achieve the control. Riedmiller reports that the swing-up and balance task required 100 iterations of the NFQ algorithm, each one requiring 1000 epochs of batch learning with the Rprop learning algorithm to train the neural net with an unspecified number $D$ of samples. This gives a total of $100,000 \times D$ sample updates. In our case, good control is obtained after approximately $60 \times 500=$ 30,000 updates, which is significantly better.

These results were obtained with the simply lo-

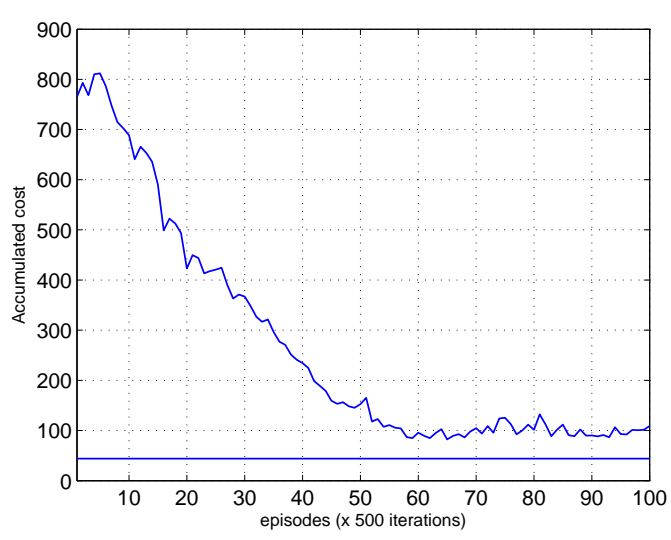

Figure 1: Average over 50 experiments of the accumulated cost, on tests of 30 seconds, with simply local updating.

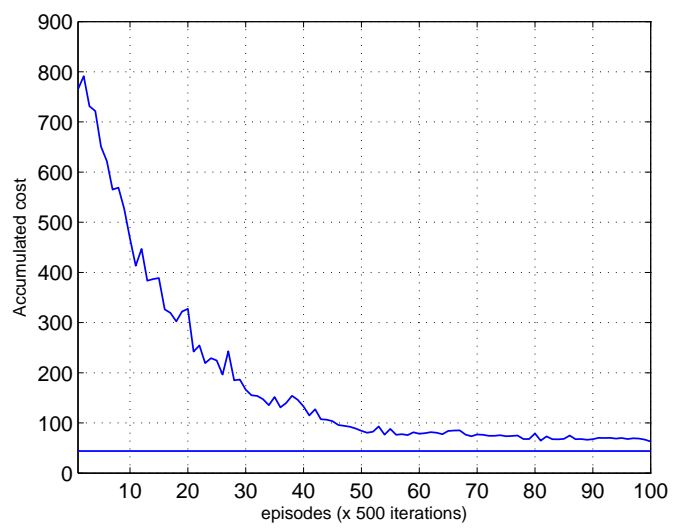

Figure 2: Average over 50 experiments of the accumulated cost, on tests of 30 seconds, with exponentially local updating.

cal updating of formula (13), which is sensible to the effect of the biased sampling. Despite being good results, we observe that the learning curve presents some fluctuations that prevent the system to converge to a value nearer to the theoretical optimum. Such fluctuations are caused by transient learning phases during which the system is not able to swing the pendulum up, until a good policy is recovered again. This is precisely the problem we anticipated: as far as the system stabilizes near a good policy, it experiences samples mostly near the optimal policy, so that the $Q$ estimation of less experienced actions degrades, and eventually, suboptimal actions gain temporary control until the system relearns their correct value. This is the reason by which we introduced the exponent $w_{t, i}$ in the update formula (18) for exponentially local updating. Its effectiveness is shown in Figure 2.

Results show that exponentially local updating achieves convergence slightly faster and with a much 


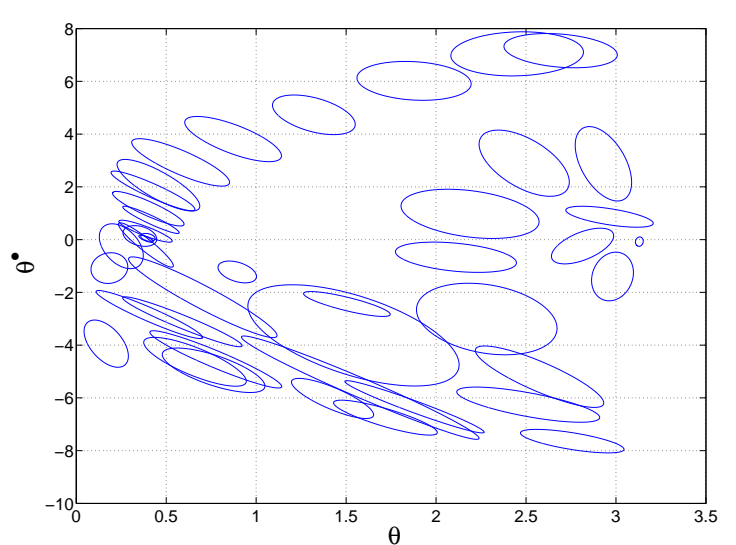

Figure 3: Distribution of Gaussians in a projection of the joint space to the state space.

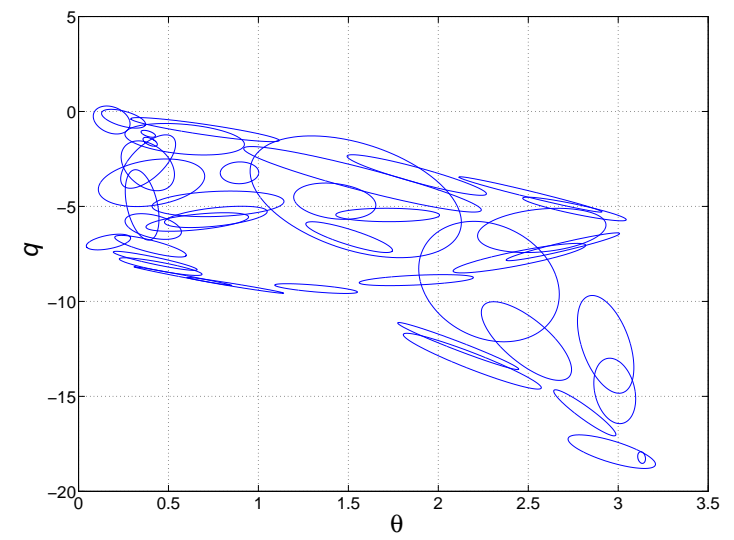

Figure 4: Distribution of Gaussians in a projection of the joint space to the $(\theta, q)$ space.

more stable behavior. In this case the average cost is reduced to near 64 , that is just 20 above the theoretical optimum, which compared with the $100-44=56$ of the simply local updating corresponds to an improvement by a factor between 2 and 3 . The number of Gaussians used in this case is also lowered to less than 60 , in average. To check the effectiveness of exponentially local updating to prevent forgetting, we observe that in the course of the 50 experiments, the system failed to swing-up the pendulum after the 50th episode only in a single occasion, compared with far more that 30 with simply local updating.

Figures 3 and 4 show two projections of the Gaussians of a typical GMM obtained for this problem after training. It can be seen that they are not equally distributed along the whole configuration space, but concentrate in the most common trajectories of the system, what constitutes an efficient use of resources.

\section{CONCLUSIONS}

We proposed a new approach for $Q$-Learning in continuous state-action spaces, in which a Gaussian Mixture Model that estimates the probability density in the joint state-action- $Q$ value space is used for function approximation. From this joint distribution we can obtain, not just the expected value of $Q$ for a given state and action, but a full probability distribution that is used to define a directed exploration-exploitation strategy. As a further benefit, from the density estimation in the joint space we can also obtain the sampling density in the state-action space. This information is used to remedy the problem of biased sampling inherent to on-line Reinforcement Learning. For this, we modified the incremental updating rule of an on-line EM algorithm in order to avoid forgetting data of less frequently sampled regions, even when exploration is reiteratively done near the goal configurations.

Tests performed on a classical RL problem, the swing-up and balance of an inverted pendulum, show that our approach improves the results of previous works when considering the number of sample updates required to achieve the goal. The comparison between our basic approach, using simply local updating, and the proposed improvement using exponentially local updating, shows that the approach is effective in reducing the perturbing effect of biased sampling.

Finally, we want to point out that the information provided by the density estimation has not been fully exploited yet. We expect to use the density information in future works to better guide exploration during learning.

\section{ACKNOWLEDGEMENTS}

This research was partially supported by Consolider Ingenio 2010, project CSD2007-00018.

\section{REFERENCES}

Arandjelovic, O. and Cipolla, R. (2005). Incremental learning of temporally-coherent gaussian mixture models. In Technical Papers - Society of Manufacturing Engineers (SME).

Bellman, R. and Dreyfus, S. (1962). Applied Dynamic Programming. Princeton University Press, Princeton, New Jersey.

Bishop, C. M. (2006). Pattern Recognition and Machine Learning (Information Science and Statistics). Springer-Verlag New York, Inc., Secaucus, NJ, USA. 
Dearden, R., Friedman, N., and Russell, S. (1998). Bayesian q-learning. In In AAAI/IAAI, pages 761-768. AAAI Press.

Dempster, A., Laird, N., Rubin, D., et al. (1977). Maximum likelihood from incomplete data via the EM algorithm. Journal of the Royal Statistical Society. Series $B$ (Methodological), 39(1):1-38.

Doya, K. (2000). Reinforcement learning in continuous time and space. Neural Comput., 12(1):219-245.

Duda, R. O., Hart, P. E., and Stork, D. G. (2001). Pattern classification. John Wiley and Sons, Inc, New-York, USA.

Engel, Y., Mannor, S., and Meir, R. (2005). Reinforcement learning with gaussian processes. In ICML '05: Proceedings of the 22nd international conference on Machine learning, pages 201-208, New York, NY, USA. ACM.

Ernst, D., Geurts, P., and Wehenkel, L. (2005). Tree-based batch mode reinforcement learning. J. Mach. Learn. Res., 6:503-556.

Figueiredo, M. (2000). On gaussian radial basis function approximations: Interpretation, extensions, and learning strategies. Pattern Recognition, International Conference on, 2:618-621.

Ghahramani, Z. and Jordan, M. (1994). Supervised learning from incomplete data via an em approach. In Proceeding of Advances in Neural Information Processing Systems (NIPS'94), pages 120-127. San Mateo, CA: Morgan Kaufmann.

Gordon, G. J. (1995). Stable function approximation in dynamic programming. In ICML, pages 261-268.

Neal, R. and Hinton, G. (1998). A view of the em algorithm that justifies incremental, sparse, and other variants. In Proceedings of the NATO Advanced Study Institute on Learning in graphical models, pages 355-368, Norwell, MA, USA. Kluwer Academic Publishers.

Nowlan, S. J. (1991). Soft competitive adaptation: neural network learning algorithms based on fitting statistical mixtures. PhD thesis, Pittsburgh, PA, USA.

Ormoneit, D. and Sen, S. (2002). Kernel-based reinforcement learning. Machine Learning, 49(2-3):161-178.

Rasmussen, C. and Kuss, M. (2004). Gaussian processes in reinforcement learning. Advances in Neural Information Processing Systems, 16:751-759.

Riedmiller, M. (2005a). Neural fitted Q iteration-first experiences with a data efficient neural reinforcement learning method. Lecture notes in computer science, 3720:317-328.

Riedmiller, M. (2005b). Neural Reinforcement Learning to Swing-up and Balance a Real Pole. In Proceedings of the 2005 IEEE International Conference on Systems, Man and Cybernetics, volume 4, pages 3191-3196.

Rottmann, A. and Burgard, W. (2009). Adaptive autonomous control using online value iteration with gaussian processes. In Proceedings of the 2009 IEEE International Conference on Robotics and Automation (ICRA'09), pages 2106-2111.
Sato, M.-A. and Ishii, S. (2000). On-line em algorithm for the normalized gaussian network. Neural Comput., 12(2):407-432.

Song, M. and Wang, H. (2005). Highly efficient incremental estimation of gaussian mixture models for online data stream clustering. In Proceedings of SPIE: Intelligent Computing: Theory and Applications III, pages 174183, Orlando, FL, USA.

Sutton, R. and Barto, A. (1998). Reinforcement Learning: An Introduction. MIT Press, Cambridge, MA.

Watkins, C. and Dayan, P. (1992). Q-learning. Machine Learning, 8(3-4):279-292. 